\title{
MAKING LECTURES COME ALIVE WITH A DATA PROJECTOR
}

\author{
J.V. RINGWOOD \\ School of Electronic Engineering, Dublin City University, Glasnevin, Dublin 9, Ireland
}

\begin{abstract}
The relatively recent advent of widely available data projectors, coupled with the availability of CAD packages for control systems with graphical input/output facilities provides a mechanism for transforming lectures into shows where students can see control systems come alive. Experiences over a range of control systems courses at Dublin City University (DCU) have been positive, where a data projector has been used over a trial period of eighteen months.
\end{abstract}

Key Words. Computer-aided instruction, computer-aided design, data projection, system theory, adaptive systems

\section{INTRODUCTION}

Data projectors, in the current context, are taken to mean devices which allow the projection of a computer screen image onto an opaque white screen. The basic objective of such a device is to give a demonstrator or instructor the facility to show an audience or student class an enlarged image of the computer screen that he/she is working on and the effects of the computer commands that he/she is issuing. In a control systems scenario, this might allow a classroom of students to follow a lecturer through the design and evaluation of a control system.

Such an educational medium is not new. However, recent improvements in the technology have dramatically improved the performance/price ratio, making a data projector a cost-effective instructional tool. A range of such devices, along with performance and price specifications, is discussed in Section 2.

At Dublin City University (DCU), this educational medium finds use on most courses related to signals, systems and control system design. In particular, the data projector is used heavily in a master's level course on Adaptive Systems in Signal Processing and Control. This course benefits greatly, since many of the computations required in the evaluation of examples are iterative, the only effective demonstration medium being through computer-based analysis.

Control systems and the study of signals and systems in general is an ideal candidate for the data projector medium. A number of wellknown CAD packages for signals and systems are available, and many real-world examples may be drawn on. In addition, many control engineering textbooks are now oriented towards $\mathrm{CAD}$ and in many cases custom software is provided to aid in the evaluation of examples.

Demonstrations of applications and examples through the use of the computer/projector combination at DCU have been found to be particularly effective. Students seem to be generally enthused with the colour, variety and dynamic movement which such a medium brings to a lecture.

\section{DATA PROJECTORS}

In order to be useful in a lecture scenario, a projector should have the capability to project an image of a computer screen onto a large screen, capable of being viewed by a large (> 40 people) audience. A similar facility could, in theory, be provided by a large VDU screen. However, devices of this type capable of generating an image of 
approx. $2 \mathrm{~m} \times 2 \mathrm{~m}$ would lack portability, an possibly be prohibitively costly. In sourcing a suitable device for the School of Electronic Engineering at DCU, effort was concentrated on a portable device, allowing its use in meetings, presentations (both internal and external) and classroom situations. Portability between classrooms was also an issue. Generally, data projectors may be divided into three broad categories:

(a) Palette-type devices

(b) Pure data projectors

(c) Video projectors

Palette-type devices consist of a rectangular frame containing a translucent LCD screen. This is placed on top of an overhead projector (OHP), and selectively attenuates the OHP image. As far as the author is aware, these represented the earliest portable data projection devices. Originally, only monochrome devices were available, but now colour devices, up to VGA quality, are available.

\section{Table 1 Comparison of device categories}

\begin{tabular}{|l|c|c|c|}
\hline & Palette & Data Proj. & Video Proj. \\
\hline Type & 1 & 2 & 3 \\
\hline Resolution & $2-1$ & $2^{2}$ & 3 \\
\hline Portability & 2 & 1 & $1 \cdot^{3}$ \\
\hline Cost & 1 & 2 & 3 \\
\hline $\begin{array}{l}\text { Main } \\
\text { Advantage }\end{array}$ & $\begin{array}{l}\text { Portabillty } \\
+ \text { cost }\end{array}$ & $\begin{array}{l}\text { Exact } \\
\text { requirement }\end{array}$ & $\begin{array}{l}\text { Extra } \\
\text { facilities }\end{array}$ \\
\hline $\begin{array}{l}\text { Main } \\
\text { Disadvan. }\end{array}$ & $\begin{array}{l}400 w \text { OHP } \\
\text { required }\end{array}$ & $\begin{array}{l}\text { Limited } \\
\text { resolution }\end{array}$ & High cost \\
\hline
\end{tabular}

Pure data projectors, unlike video projectors accept VGA-type output only. Video projectors, on the other hand, accept RGBtype inputs and sometimes have an auxiliary data input as well. If not, a converter may be used to modify the VGA signal to RGB format. While providing the extra facility for video projection, video projectors are generally more expensive, and if a data projection only facility is required, incurs unnecessary expense. Video projectors, however, generally offer better resolution, with horizontal scanning rates up to $80 \mathrm{kHz}$,

\footnotetext{
1Pallette devices have generally got poorer resolution than pure data projectors, but some can do up to VGA.

${ }^{2}$ Currently only VGA is available, but an SVGA device is due shortly.

${ }^{3}$ If a converter is requires, portability is impaired.
}

while a maximum rate of $35 \mathrm{kHz}$ (< SVGA) is available on pure data projectors.

Table 1 summarises the relative merits of each category of device. Generally, it has been found that pure data projectors offer the best compromise for classroom use. The following table reviews a number of machines (not an exclusive list) and gives an estimate of cost at Irish prices in US\$ (excluding sales tax).

\section{Table 2 Sample of available data projectors}

\begin{tabular}{|l|l|l|l|l|}
\hline Make & Model & Type $^{4}$ & Quality $^{6}$ & Price $^{6}$ \\
\hline Barco & 300 & 2 & VGA & 9000 \\
\hline Barco & 600 & 3 & SVGA+ & 13500 \\
\hline GE & 310 & 3 & SVGA & 13000 \\
\hline Sanyo & PLC300PB & 3 & VGA & 13500 \\
\hline Sayett & 7799 & 2 & VGA & 12000 \\
\hline Sharp & XG3800E & 3 & VGA & 12000 \\
\hline Sharp & $1050^{7}$ & 1 & VGA & 8000 \\
\hline
\end{tabular}

In addition to the information shown in Table 2, there is a wide diversity in extra features on each device. Some have motorised focus and interchangeable lenses, some have remote control and some have built in 'disk-play' devices. Also, Table 2 concentrates only on portable devices; a wide range of ceiling mount projectors are also available. A comparison of a subset of the devices listed above is given in Tomalski (1992).

Experience in choosing a data projector has shown that, in spite of specification similarities, there can be a significant difference in projection quality and brightness between devices, which can only be ascertained by means of side-by-side testing.

\section{PROJECTOR USE AT DCU}

In the School of Electronic Engineering at Dublin City University, use of the data projector as a lecturing aid spans a number of courses. As stated previously, these courses are generally involved with some aspect of signals or control systems, but it is

\footnotetext{
${ }^{4}$ Refer to Table 1.

${ }^{5}$ This is given in terms of the resolution, which is determined by the maximum horizontal scan rate.

${ }^{6}$ Includes the cost of a computer to RGB converter, where appropriate.

${ }^{7}$ Requires a $400 \mathrm{w}$ OHP.
} 
also used extensively in courses on software engineering.

\subsection{Hardware and Software}

A standard projection rig has been set up which facilitates fast set-up times and ease of use. This consists of a two tier trolley/stand with the top platform having a tilt adjustment in two dimensions. The projector occupies this platform, with the lower level reserved for a 486 notebook $P C$, as shown in Fig.1. The PC sees the projector as an external monitor, but the colour LCD display on the notebook PC remains active. This feature is important, since it allows the lecturer to face the class and divide his/her attention between the class and the monitor easily. Unfortunately, many notebook PCs blank the LCD screen when an external monitor is detected.

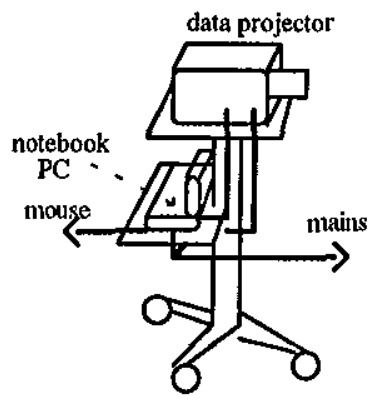

Fig.1. Trolley with projector and PC

For control systems courses, the principal tool is the MATLAB/SIMULINK suite, which is run in the Windows environment. This is complemented by a number of the associated toolboxes, including the Signal Processing and the Control Systems Toolbox. In addition, a custom toolbox, containing custom-built MATLAB macros and functional blocks, is also mounted.

\subsection{Control Systems Courses at DCU}

In the four year BEng programme in Electronic Engineering at DCU, the following courses are available:

(a) System Dynamics I, Year 2, compulsory.

(b) System Dynamics II, Year 3, compulsory.

(c) Control Core, Year 4, compulsory.

(d) Digital Control Option, Year 4, optional.

In addition, a number of control-related modules are available on the MEng course in
Electronic Systems. The author currently teaches on courses (b) and (d) above and a course on Adaptive Systems in Signal Processing and Control in the MEng. The data projector is used on each of these courses, with utilisation highest on the MEng course and the Digital Control Option. Examples of the type of use to which the computer/projector set is put are given in Section 5.

\section{THE ROLE OF COMPUTER-ASSISTED INSTRUCTION IN CONTROL SYSTEMS}

The general approach in the presentation of control systems techniques roughly follows the following steps:

- Presentation of the theory associated with the technique,

- Evaluation of an illustrative example,

- Review of performance and examination of practical additions.

Computer-aided presentation has been found to be particularly effective for the last two stages outlined above. It allows multiple examples to be evaluated over a wide range of operating conditions, proving beyond doubt the validity of the theory. It also provides a facility for immediate and graphic answers to 'what-if' type student questions.

An increasingly popular feature of modern control systems textbooks is the production of a set of computer programs or subroutines to accompany the text. These include titles from authors such as Phillips and Nagle (1990), Thompson (1989), Thaler (1989), Golten and Verwer (1991), Franklin et al (1990), Dorf (1992) and Bishop (1993). In some cases, a set of computer program listings are provided (for example, in Thompson (1989)), others provide a disk containing a dedicated software package (e.g. in Thaler (1989), Golten and Verwer (1991) and Dorf (1992)) while other authors provide the software as an optional extra (as in Phillips and Nagle (1990) and Franklin (1990)). The text by Franklin et al (1990) has the interesting feature that the software associated with the text is a set of MATLAB M-files, which complement those contained in the MATLAB Control Systems Toolbox. Finally, the text by Bishop (1993) is based around MATLAB and gives all the MATLAB macro code in the text associated with each example. This book is a companion to Dorf (1992). 
These books generally provide either general CACSD software or software which may be used to illustrate the examples in the text, in which case code is often included to provide plots, etc. Such material provides easy entry to computer usage during classroom sessions, for even if one of the above texts is not being followed, examples relevant to the course (with supporting software) may be extracted. This methodology incurs a small overhead in preparation time.

\section{EXAMPLES}

Within the courses specified in Section 3.2, the data projector/PC combination is used for a wide variety of demonstrations. Some particularly effective ones are outlined in the following sections:

\subsection{PID Parameter Tuning}

A classical feedback loop is set up with a conventional three-term controller in the forward loop. With multiple simulation runs, students may clearly see the effects on the time response of variations in each of the controller parameters. Also, the addition of effects such as control signal saturation, output noise and square-wave setpoints provide clear motivation for the practical additions of anti-integral windup, derivative filtering and derivative on output only, respectively

\subsection{Optimal Controller Calculations}

Using SIMULINK, a simple state-feedback loop is set up. In the background, MATLAB provides a recursive solution to the matrix Ricatti equation, allowing the lecturer to demonstrate the variation in feedback gains, closed-loop poles and the responses of state and control signals according to the choice of state and control weighting matrices.

\subsection{Adaptive PID Controller}

Fig.2 shows a phase-margin explicit adaptive PID controller utilising recursive least squares for identification. Using this set-up, responses may be demonstrated for variations in:

- Forgetting factor
- Initial covariance scaling

- Initial model parameter estimates

- Order of plant model

- Identification algorithm (e.g. LMS)

- Setpoint signal

- Phase margin specification

- Actual plant parameters

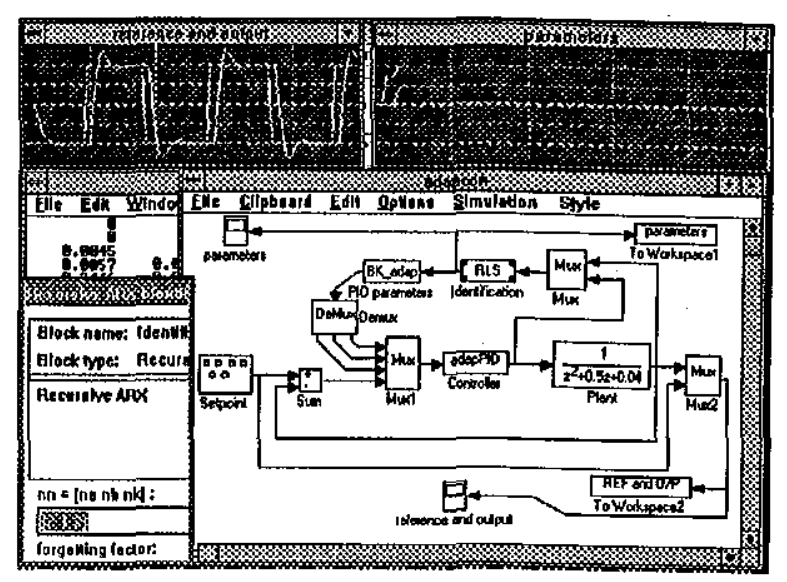

Fig.2. Adaptive PID controller demonstration

\section{CONCLUSIONS}

The inclusion of computer-aided presentation in control systems lecturers has been well received by the students. The extra overhead in terms of preparation and set-up has been more than compensated for in teaching effectiveness.

\section{REFERENCES}

Bishop, R.H. (1993). Modern Control System Analysis and Design Using MATLAB ${ }^{\circledR}$, Addison Wesley.

Dorf, R.C. (1992). Modern Control Systems (6th Ed.), Addison Wesley.

Franklin, G.F., J.D. Powell and M.L. Workman (1990). Digital Control of Dynamic Systems (2nd Ed.), Addison Wesley.

Golten, J. and A.Verwer (1991). Control System Design and Simulation, McGrawHill.

Phillips, C.L. and H.T. Nagle (1990). Digital Control System Analysis and Design (2nd $E d$.$) , Prentice Hall.$

Thaler, G.J. (1989). Automatic Control Systems, West Publishing.

Thompson, S. (1989). Control Systems Engineering and Design, Longman.

Tomalski, R. (1992). New age crystal cinema, What Video, April. 